\title{
Biophysical and Microstructural Changes of Swelling Cornea Caused by Endothelial Cells Damage
}

\author{
Fengying $\mathrm{HE}^{1,2 *}$, Jian $\mathrm{LIU}^{3 *}$, Panpan YE ${ }^{1}$, Jing WU ${ }^{1}$, Guanlu LIANG ${ }^{3}$, Wen XU ${ }^{1}$ \\ * These authors contributed equally to this work. \\ ${ }^{1}$ Eye Center, Second Affiliated Hospital of Zhejiang University, Hangzhou, China, ${ }^{2}$ Department of \\ Ophthalmology, Huzhou Central Hospital, Huzhou, China, ${ }^{3}$ Department of Ophthalmology, \\ Zhejiang Hospital, Hangzhou, China
}

Received August 27, 2018

Accepted May 7, 2019

Epub Ahead of Print August 19, 2019

\begin{abstract}
Summary
Biophysical properties and microstructural changes of swelling cornea which caused by endothelial cells damage will be evaluated. Swelling cornea models were established by endothelial cells damage in 114 Sprague Dawley rats. Relative gray value, swelling rate and light transmittance were measured to evaluated the biophysical properties and microstructure changes were observed by transmission electron microscopy. Relative gray value decreased while swelling rate rose along with time and both of them reached relative stability after 7 days. Light transmittance showed a decline trend with time even after corneal thickness had reached stable stage. Observed by transmission electron microscopy, interfibrillar distance increased, fewer proteoglycans coating appeared and remnants proteoglycan branches became thinner and longer in 7 days. Diameter of fibrils didn't change obviously with time. In cornea edema models caused by endothelial cells damage, the changes of biophysical property and microstructure can help us evaluate corneal edema accurately and objectively.
\end{abstract}

\section{Key words}

Swelling cornea • Biophysical properties • Microstructure

\section{Corresponding author}

W. Xu, Eye Center, Second Affiliated Hospital of Zhejiang University, College of Medicine, Jiefang Road 88, Hangzhou, 310009, China. E-mail: xuwenhz2002@aliyun.com

\section{Introduction}

As the frontmost structure of the eyeball, cornea is the primary refractive element when external light enters the eyeball. Under normal circumstances, cornea is in a state of relative dehydration. Maintaining corneal deturgescence is essential for keeping transparency. When it is in pathological conditions and increases water content, cornea will lose its transparency. As a result, the vision will be severely affected. Comprising over $90 \%$ of corneal thickness, corneal stroma is made up mainly of collagen fibrils. They regularly pack with a highly ordered hierarchical organization and have a homogeneous diameter of $25-30 \mathrm{~nm}$ in human (Bancelin et al. 2014, Chen et al. 2015). It is known that in swelling corneas, the interfibrillar distance increased as well as overall lamellas disorganized (Muller et al. 2001). However, the biophysical properties and microstructural features were seldom analyzed simultaneously.

In this study, we established corneal edema model by endothelium damaged in rats. Using ultrasonic corneal thickness measurement technology, anterior segment photography, light transmission measurement and transmission electron microscope (TEM), we aim to display the biophysical properties and microstructural changes in swelling corneas. 


\section{Material and Methods}

\section{Sample preparation}

The study was conducted according to guidelines approved by the Ethical Committee in Animal Research at Zhejiang University and the tenets of the NIH Statement for the Use of Animals in Research. Five-week-old Sprague Dawley (SD) rats with body weight ranging from 130 to $150 \mathrm{~g}$ were pre-checked for corneal disease before obtained samples. They were housed in the animal labs at Zhejiang University for one week prior to use. After the rats sacrificed with intraperitoneal injection of overdosed anesthetic of chloral hydrate, eyeballs were obtained with epithelium intact immediately. A circle with a radius of $2 \mathrm{~mm}$ on the central corneal endothelium was stripped by a needle. Then, the eyeball was totally immersed in the Ringer's solution (sodium chloride: $0.85 \%$; potassium chloride: $0.03 \%$; calcium chloride: $0.033 \%$ ) for $0 \mathrm{~h}, 2 \mathrm{~h}, 4 \mathrm{~h}, 8 \mathrm{~h}$, 1 day, 2 days, 4 days, 7 days, 8 days and 9 days. The eyeballs were stored at $4{ }^{\circ} \mathrm{C}$ for incubation and the solution was replenished every $24 \mathrm{~h}$ to maintain its composition.

\section{Anterior segment photography and relative gray value}

Six eyeballs were performed anterior segment photography at each time point. To evaluate the opacity quantitatively and objectively, the relative gray value (RGV) was performed as described previously (Xu et al. 2005). Briefly, corneas were excised along cornea-scleral limbus and put onto a white paper with black crosses. Photos were took with same camera exposure settings and illumination conditions and then scanned them and obtained the data using IPP software (Image Pro Plus 6.0).

$$
\mathrm{RGV}=\frac{\text { grey value of black cross }}{\text { mean grey value of four adjacent white background }}
$$

\section{Central corneal thickness and swelling rate}

Central corneal thickness (CCT) were measured at each time point by ultrasound pachymeter (SP-3000, TOMEY, Japan). Each measurement consisted of 10 consecutive readings and the average was calculated after excluding the highest and lowest readings. To account for differences of corneal thickness between samples, swelling rate (SR) rather than CCT was evaluated.

$$
\mathrm{SR}=\frac{\text { change of } C C T}{\text { baseline } C C T} * 100 \%
$$

\section{Light transmission measurement}

Six corneas were cut with a $10-\mathrm{mm}$ trephine at each time point. The cornea was washed 3 times using phosphate buffer solution (PBS) and placed on a 48-well microplate. Blank-corrected light absorbance was measured with microplate reader (Multiskan Spectrum, Thermo Scientific, USA) with wavelengths ranged from $400 \mathrm{~nm}$ to $800 \mathrm{~nm}$. Light transmittance (LT) was calculated by the Beer-Lambert equation:

Transmittance $(\%)=10^{2 \text {-absorbance }}$.

\section{Transmission electron microscopy}

Corneas at 0 day, 1 day, 2 days, 4 days, 5 days, 6 days, 7 days and 14 days were fixed in $2.5 \%$ glutaraldehyde overnight. Each specimen from central corneas were cut with a 3-mm trephine and rinse with PBS. Stained the samples with $1 \%$ osmium tetroxide on ice for $1 \mathrm{~h}$ and rinsed again. After dehydration with a graded series of ethanol solutions, the samples were embedded in Eponate 12 (Ted Pella, Redding, CA) at $60{ }^{\circ} \mathrm{C}$ for 2 days. Sections were cut by an ultramicrotome (Ultracut UCT; Leica, Austria) and stained with uranyl acetate. Then they were examined using a TEM (Philips TECNAL-10, Japan).

\section{Statistical analysis}

The data were analyzed by SPSS 20.0 statistical package. The normality of distribution of continuous variables was tested by one-sample KolmogorovSmirnov test. Continuous variables with normal distribution were presented as mean \pm standard deviation (SD) and analyzed by Analysis of Variance (ANOVA). The Bonferroni test was used when the variances were equal, and the Tamhane's T2 test was used when the variances were unequal. Non-normal variables were analyzed by Kruskal-Wallis test. A value of $\mathrm{P}<0.05$ was considered to be statistically significant.

\section{Results}

Anterior segment photography and relative gray value

As the immersing time prolonged, corneal oedema was aggravated (Fig. 1A). At 0 day, the corneas were transparent and the iris texture can be seen clearly. From 1 day to 7 days, the corneas gradually became opaque and edematous. At the time of 1 week, corneas were severely edematous and iris was invisible.

Mean RGVs of swelling corneas at different time ( 0 day, 2 h, 4 h, 8 h, 1 day, 2 days, 4 days, 7 days, 
8 days and 9 days) were $68.04 \pm 3.94,64.42 \pm 3.31$, $60.58 \pm 0.39, \quad 58.62 \pm 1.14, \quad 54.54 \pm 1.81, \quad 52.86 \pm 4.04$, $49.68 \pm 2.22,39.52 \pm 3.88, \quad 36.35 \pm 3.43$ and $38.72 \pm 3.69$, respectively. It plunged from 0 day to 1 day, and reached relatively steady state after 7 days. (Fig. 1B).
A

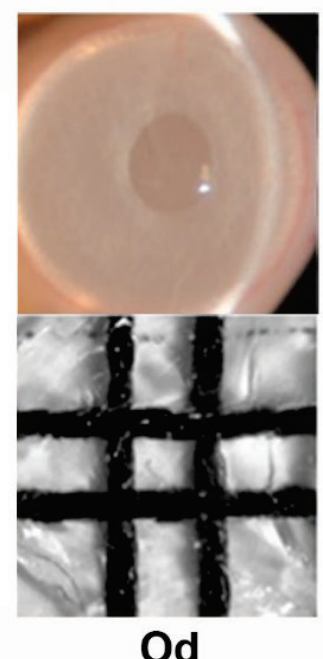

B

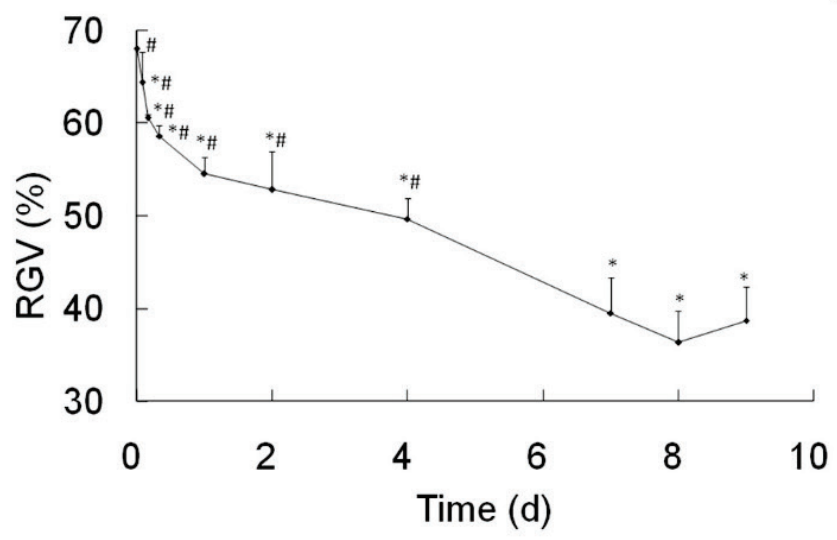

D

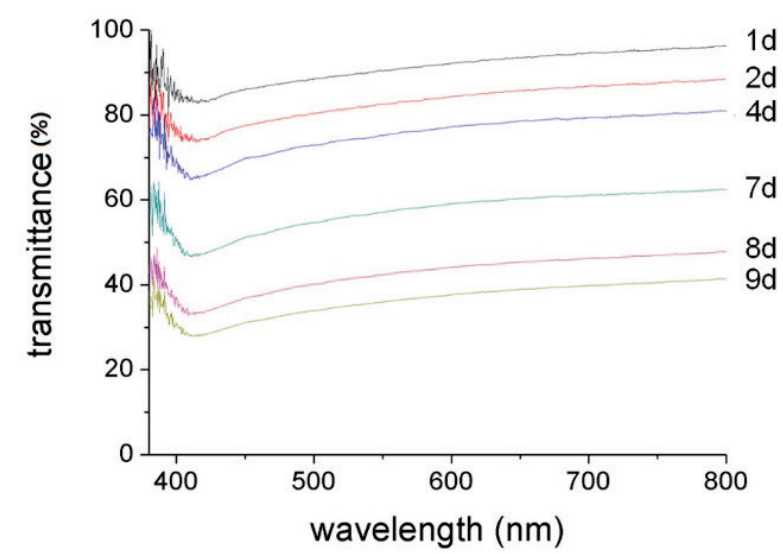

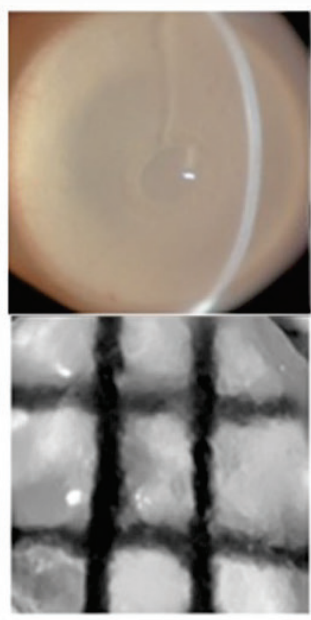

4d

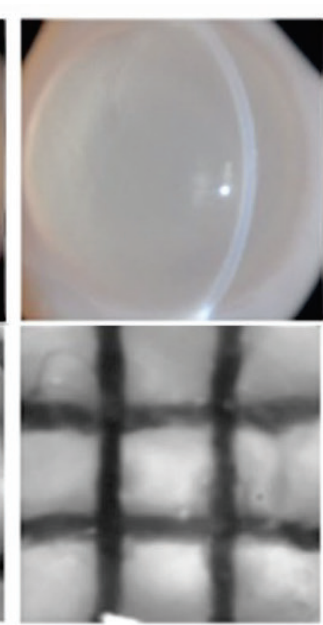

7d

C

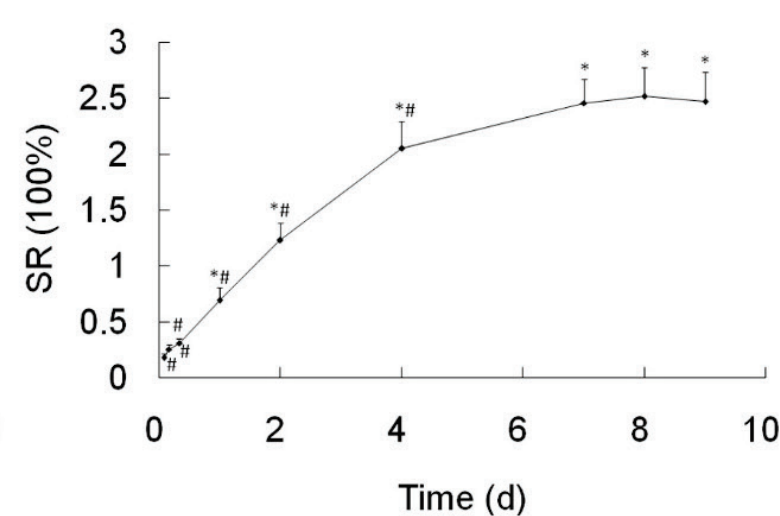

E

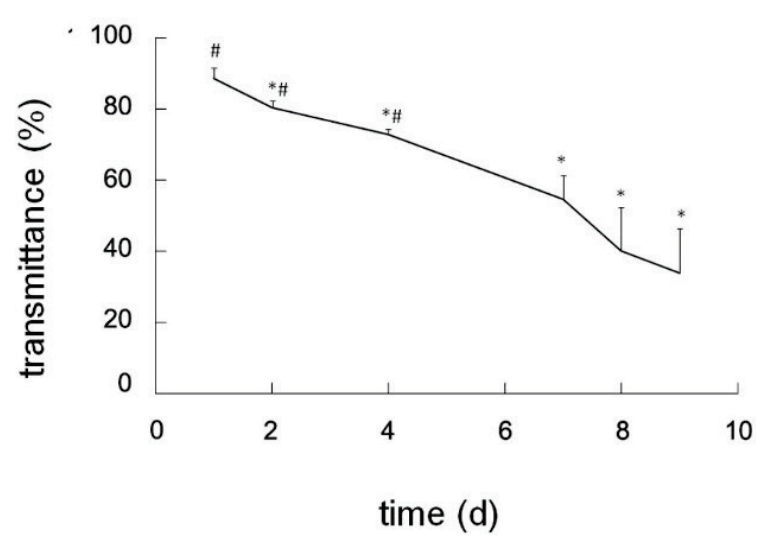

Fig. 1. Biophysical changes of swelling cornea caused by endothelial cells damage at different times. (A) anterior segment photography; (B) RGV, ${ }^{*} \mathrm{P}<0.05$ compared with 0 day, ${ }^{\#} \mathrm{P}<0.05$ compared with 9 days; (C) SR, ${ }^{*} \mathrm{P}<0.05$ compared with $2 \mathrm{~h}$, ${ }^{\#} \mathrm{P}<0.05$ compared with 9 days; (D) LTs of swelling corneas with different wavelengths; (E) green light transmission of swelling corneas, ${ }^{*} \mathrm{P}<0.05$ compared with $2 \mathrm{~h},{ }^{\#} \mathrm{P}<0.05$ compared with 9 days. Data are mean $\pm \mathrm{SD}$. 


\section{Swelling rate}

To reflect the changes of corneal thickness exactly, SRs were analyzed in this study. Mean SRs of swelling corneas at different time $(2 \mathrm{~h}, 4 \mathrm{~h}, 8 \mathrm{~h}, 24 \mathrm{~h}$, $48 \mathrm{~h}, 4$ days, 7 days, 8 days and 9 days) were $18.08 \pm 4.04 \%, \quad 25.47 \pm 4.31 \%, \quad 31.12 \pm 4.24 \%$, $69.21 \pm 11.38 \%, \quad 122.84 \pm 15.62 \%, \quad 204.83 \pm 24.51 \%$, $245.50 \pm 21.22 \%, 251.45 \pm 26.00 \%$ and $246.86 \pm 26.50 \%$. Opposite to RGV, SR rose rapidly from the start, slowed down at 4 days and reached steady state at 7 days. (Fig. 1C).

\section{Light transmission}

The LTs of swelling corneas declined throughout the visible light range as time went by, and rose slightly with the increase of wavelengths (Fig. 1D). Since different lights had similar trends, LTs at $500 \mathrm{~nm}$ wavelength (green light) were measured. The results showed LTs after 2 days had significant difference compared with 1 day $(\mathrm{P}<0.05)$. Besides, compared with 9 days, the values before 4 days were statistically different (Fig. 1E).

\section{Ultrastructure of the stroma}

Cross sectioned and longitudinally sectioned collagen bundles of the central region of swelling corneas were observed by TEM. Regular arrangement of collagen fibrils within a lamellar was observed in fresh corneas. In early stage of corneal edema (within 1 day), the basic structure of collagen fibrils was intact and the collagen fibers within a lamellar were in a regular arrangement. During the chronic phase or late stage (from 1 day to 7 days), the overall structure became irregular gradually. Not only interfibrillar distance increased, but the collagen fibrils themselves appeared different shapes, such as shorter, irregular and more disorganized, as well as necrosis cell debris appeared. In addition, the broken collagen fibrils within a lamellar were in extremely different orientations, which differed from the previous orderly arrangement situation (Fig. 2A). On the other hand, collagen fibrils coated with proteoglycans arranged closely and tethered by short and thick branches of proteoglycans in early stage. However, fewer proteoglycans coating appeared and the remnants proteoglycan branches became thinner and longer with time (Fig. 2B). The density and diameter of collagen fibrils of swelling corneas were calculated in TEM photos. The result indicated that the density dropped with time and stabilized after 6 days (Fig. 2C). However, the diameter of collagen fibrils didn't change apparently over time (Fig. 2D).

The optical properties and microstructure of swelling cornea could be scanned from Figure 3.

\section{Discussion}

The corneal endothelium plays a key role in maintaining the dehydration of cornea through 'pumpleak' mechanism. After we destroyed the endothelial cells, the pumps were not able to work properly. As a consequence, the balance between fluid absorption and exclusion was disturbed.

In this article, the transparency of the cornea decreased significantly and the iris texture turned into invisible gradually over time. RGV plunged within 1 day, then slowed down and reached a relatively stable stage after 7 days. Changes in corneal thickness are very sensitive to corneal edema (Monti et al. 2002). In our study, SR increased rapidly within 4 days, then slowed down and stabilized after 7 days. These results showed that RGV had close relationship with SR. Interestingly, from beginning to 4 days, trends of these two parameters were not completely reversed. This indicated there were other factors participated in the process of corneal opacity except changes of corneal thickness.

LT is an important optic parameter for corneal tissue. In general conception, collagen fibrils were regularly packed and arranged as orthogonal layers or lamellae. This lattice-like structure produces minimal light scattering, or maximal LT, which is necessary for corneal transparency (Benedek 1971, Farrell et al. 1973, Maurice 1957). Once corneal edema occurs, swelling corneas will show remarkable periodic fluctuations in the refractive index over distances comparable to, or larger than one half the wavelength of light, which will be enough to increase light scattering. Change of refractive index was always accompanied by the changes in corneal ultrastructure. The existence of a large number of keratocytes in the stroma will make scattering problem even more perplexing. Keratocytes are the main cells in the corneal stroma. They are transparent except for their nuclei and contribute up to $15 \%$ of the total stromal volume (Huang and Meek 1999). Without any doubt, when cornea swells to some extent, keratocytes may form 'lakes', which bring great light scattering (McCally et al. 2007, Moller-Pedersen et al. 2000). This process was supposed to have relationship with the loss of protein expression or development of prominent actin filament 
bundles (Jester et al. 2005). Meek found that local fibrils disordering, refractive index mismatch and corneal thickness increasing together accounted for $20 \%$ increase in light scattering and additional scattering was probably caused by 'lakes' (Meek et al. 2003). Those may explain why we found LTs decreased with time even after SR had reached stable stage in this research.
A
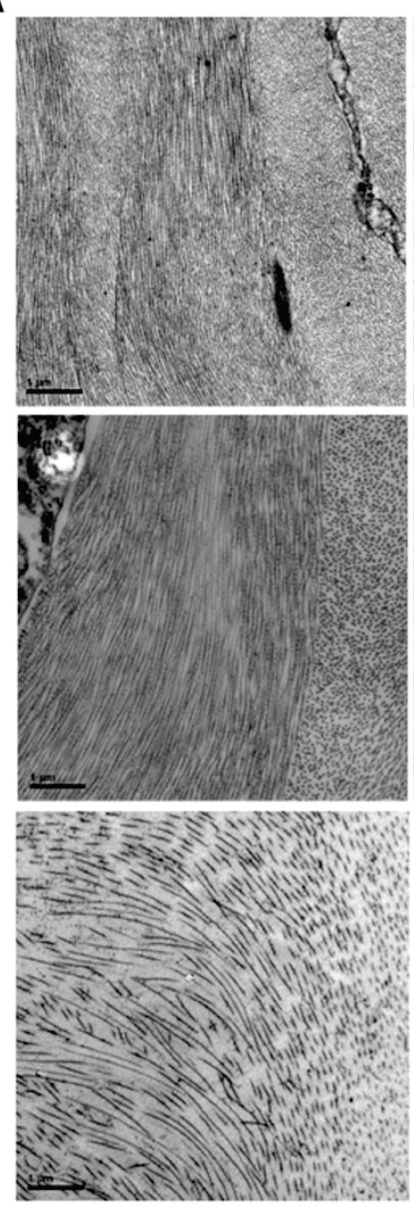

C

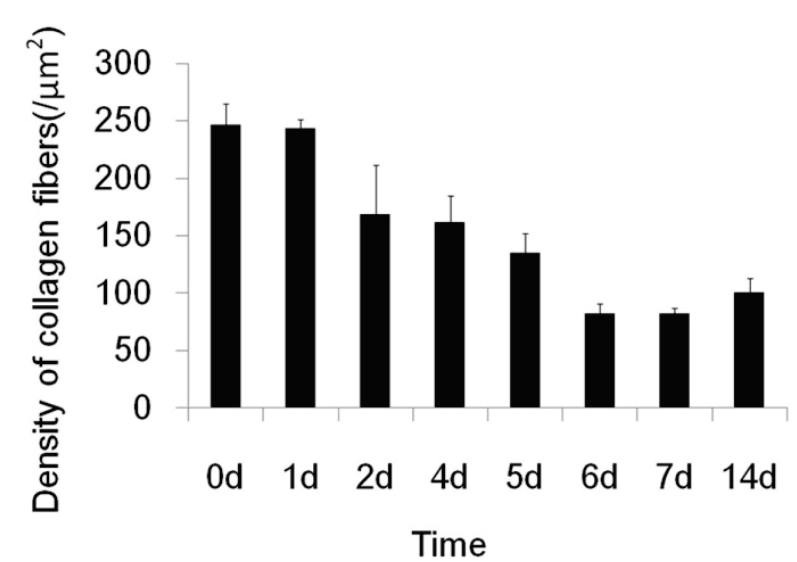

B

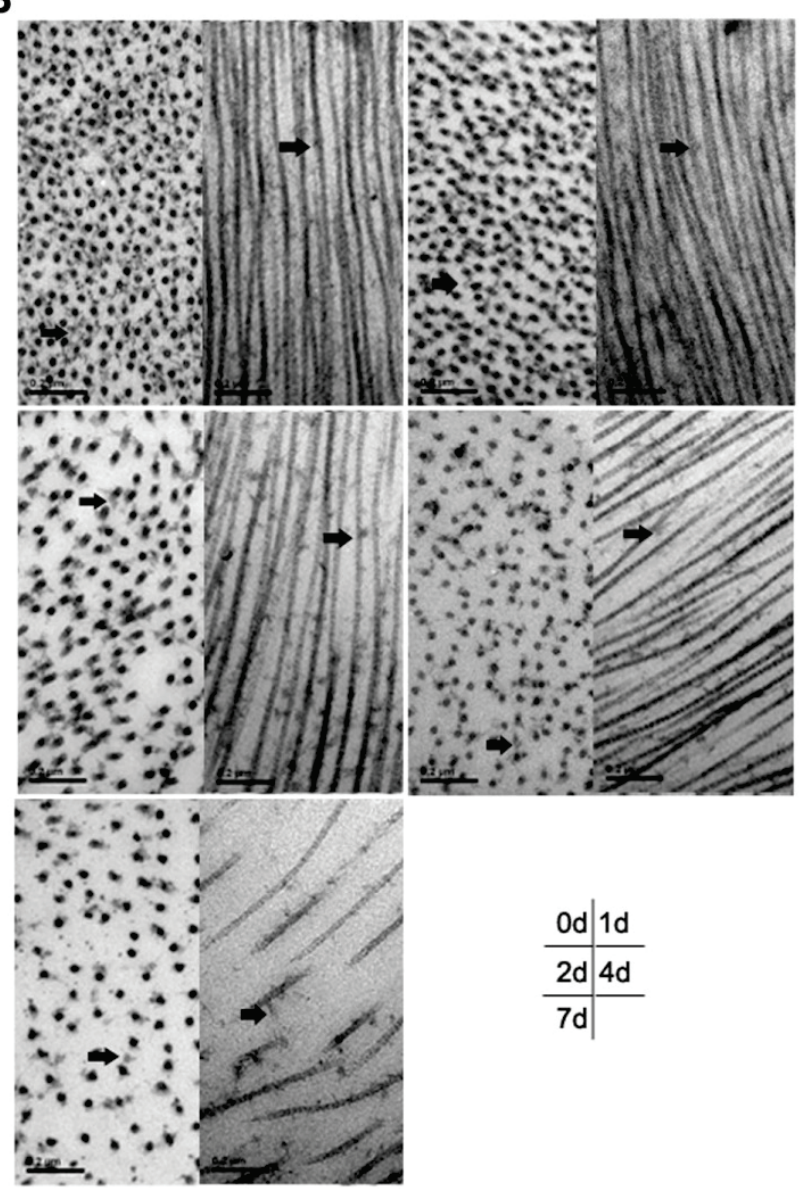

D

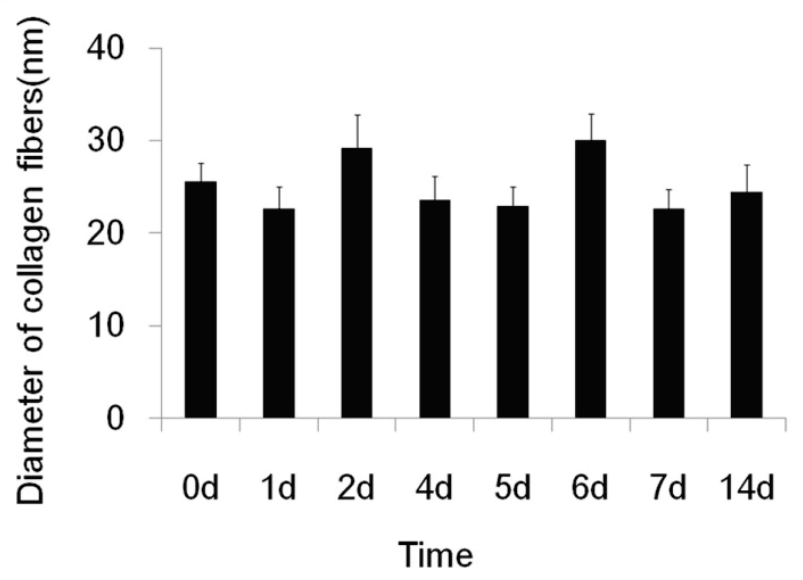

Fig. 2. TEM of corneal stroma in swelling corneas. (A) Ultrastructure became irregular and disorganized. The bars represent $1 \mu \mathrm{m}$ (20000x magnification); (B) Cross sectioned (left) and longitudinally sectioned (right) collagen fibrils were observed over time. Proteoglycans in interfibrillar space were showed by black arrows. The bars represent $0.2 \mu \mathrm{m}$ (100 000x magnification); (C) Density of collagen fibrils in swelling corneas; (D) Diameter of collagen fibrils in swelling corneas. 


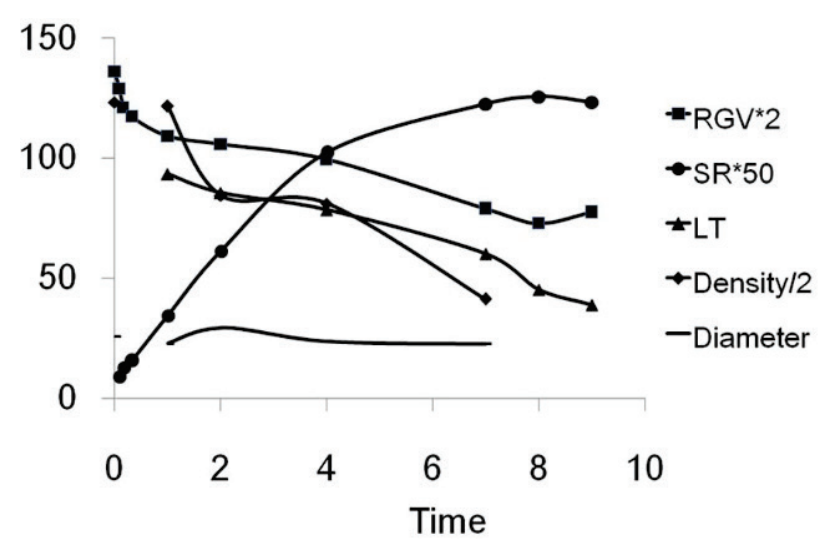

Fig. 3. Changes of biophysical properties and microstructures of swelling cornea.

Considering collagen density of corneas varies in different regions and depth of the cornea, we observed all specimen from central corneas (Boote et al. 2003, Freund et al. 1995). As TEM showed, the overall structure became irregular gradually with time increased. Not only the density of collagen fibrils and proteoglycans dropped, but also the morphology of proteoglycans changed and fewer proteoglycan coating left. Fratzl and Draxer (1993) suggested the fibrils start to be dehydrated after coating released all water in the two-stage drying model of cornea. Cheng and Pinsky (2013) pointed out that the proteoglycans attached to collagen fibrils play an important role in maintaining the order of the lattice, which is essential to keep corneal transparency. Lewis et al. (2010) proposed mechanism on how the interfibrillar distances are maintained by proteoglycans. The distances between adjacent collagen fibrils are a consequence of the balancing of two opposing forces acting on the fibrils, which the repulsive forces between the fibrils provided by the pressure exerted by water molecules and the attractive forces provided by proteoglycans.

In our research, the destruction of endothelial cells led to corneal overhydration, which disrupted the equilibrium of adjacent fibrils. We observed the density and morphology of proteoglycans changed notably over time, which may contribute to the change of corneal transparency. It is noteworthy that the electron microscopic images did not show significant differences in diameter of the collagen fibrils. Similar results were discerned in Müller's study (Muller et al. 2001). In this study, Müller made corneal stroma in extreme hydration by deionized water treatment. He found the diameter of the collagen fibrils did not be affected over time even after 6 months. Vitro experiments showed that the type $\mathrm{V}$ collagen molecule terminal domain was necessary to regulate the diameters of the formed fibrils (Birk et al. 1990). Moreover, additional mechanisms such as microfibrillar coiling and collagen-bound disaccharides also contribute to fibril diameter regulating (Meek and Knupp 2015). It seems that fibril diameter would not affected by overhydration of stroma.

We found several properties and ultrastructure changes of the swelling corneas induced by ECs-damaged in rats. Rat corneas are different with the human corneas because its endothelial cells can proliferate after mechanical injury. However, we thought it had little influence to the outcomes because the enucleated eyeballs stored at relative low temperature. In further studies, expanding sample size and in vivo studies will be considered.

\section{Conclusions}

In this study, we explored the changes among general structure, SR, optical properties and ultrastructure changes of the swelling corneas induced by ECs-damaged. The internal relationship between biophysical and microstructural changes may contribute to evaluating corneal microstructure and grade corneal edema accurately and objectively.

\section{Conflict of Interest}

There is no conflict of interest.

\section{Acknowledgements}

This research was supported by the key project of Science and Technology Department of Zhejiang Province under Grant No. 2017 C03046.

\section{References}

BANCELIN S, AIME C, GUSACHENKO I, KOWALCZUK L, LATOUR G, CORADIN T, SCHANNE-KLEIN MC: Determination of collagen fibril size via absolute measurements of second-harmonic generation signals. Nat Commun 5: 4920, 2014.

BENEDEK GB: Theory of transparency of the eye. Appl Opt 10: 459-473, 1971. 
BIRK DE, FITCH JM, BABIARZ JP, DOANE K J, LINSENMAYER TF: Collagen fibrillogenesis in vitro: interaction of types I and V collagen regulates fibril diameter. J Cell Sci 95: 649-657, 1990.

BOOTE C, DENNIS S, NEWTON RH, PURI H, MEEK KM: Collagen fibrils appear more closely packed in the prepupillary cornea: optical and biomechanical implications. Invest Ophthalmol Vis Sci 44: 2941-2948, 2003.

CHEN S, MIENALTOWSKI MJ, BIRK DE: Regulation of corneal stroma extracellular matrix assembly. Exp Eye Res 133: 69-80, 2015.

CHENG X, PINSKY PM: Mechanisms of self-organization for the collagen fibril lattice in the human cornea. $J R$ Soc Interface 10: 20130512, 2013

FARRELL RA, MCCALLY RL, TATHAM PE: Wave-length dependencies of light scattering in normal and cold swollen rabbit corneas and their structural implications. J Physiol 233: 589-612, 1973.

FRATZL P, DAXER A: Structural transformation of collagen fibrils in corneal stroma during drying. An x-ray scattering study. Biophys $J$ 64: 1210-1214, 1993.

FREUND DE, MCCALLY RL, FARRELL RA, CRISTOL SM, L'HERNAULT NL, EDELHAUSER HF: Ultrastructure in anterior and posterior stroma of perfused human and rabbit corneas. Relation to transparency. Invest Ophthalmol Vis Sci 36: 1508-1523, 1995.

HUANG Y, MEEK KM: Swelling studies on the cornea and sclera: the effects of $\mathrm{pH}$ and ionic strength. Biophys $J$ 77: 1655-1665, 1999.

JESTER J V, BUDGE A, FISHER S, HUANG J: Corneal keratocytes: phenotypic and species differences in abundant protein expression and in vitro light-scattering. Invest Ophthalmol Vis Sci 46: 2369-2378, 2005.

LEWIS PN, PINALI C, YOUNG RD, MEEK KM, QUANTOCK AJ, KNUPP C: Structural interactions between collagen and proteoglycans are elucidated by three-dimensional electron tomography of bovine cornea. Structure 18: 239-245, 2010.

MAURICE DM: The structure and transparency of the cornea. $J$ Physiol 136: 263-286, 1957.

MCCALLY RL, FREUND DE, ZORN A, BONNEY-RAY J, GREBE R, DE LA CRUZ Z, GREEN WR: Lightscattering and ultrastructure of healed penetrating corneal wounds. Invest Ophthalmol Vis Sci 48: 157-165, 2007.

MEEK KM, KNUPP C: Corneal structure and transparency. Prog Retin Eye Res 49: 1-16, 2015.

MEEK KM, LEONARD DW, CONNON CJ, DENNIS S, KHAN S: Transparency, swelling and scarring in the corneal stroma. Eye (Lond) 17: 927-936, 2003.

MOLLER-PEDERSEN T, CAVANAGH HD, PETROLL WM, JESTER JV: Stromal wound healing explains refractive instability and haze development after photorefractive keratectomy: a 1-year confocal microscopic study. Ophthalmology 107: 1235-1245, 2000.

MONTI D, CHETONI P, BURGALASSI S, NAJARRO M, SAETTONE MF: Increased corneal hydration induced by potential ocular penetration enhancers: assessment by differential scanning calorimetry (DSC) and by desiccation. Int J Pharm 232: 139-147, 2002.

MULLER LJ, PELS E, VRENSEN GF: The specific architecture of the anterior stroma accounts for maintenance of corneal curvature. Br J Ophthalmol 85: 437-443, 2001.

XU W, YAO K, WANG K, SUN Z, DAI N: Change of protein and cell in oxidative cataract. Ophthalmic Res 23: 135-138, 2005. 